\title{
Carga de Mortalidad Asociada a la Inactividad Física en Bogotá
}

\author{
Felipe Lobelo ${ }^{1}$, Rusell Pate ${ }^{2}$, Diana Parra ${ }^{3}$, John Duperly ${ }^{4}$ y Michael Pratt ${ }^{5}$ \\ ${ }^{1}$ Medico. Ph. D (Candidato). Departamento de Ciencias del Ejercicio, Facultad de Salud \\ Publica, Universidad de Carolina del Sur. 730 Devine Street, Columbia, SC, 29208. E- \\ mail: lobelo@mailbox.sc.edu \\ ${ }^{2}$ Exercise Science. PT. Ph. D. Departamento de Ciencias del Ejercicio, Facultad de \\ Salud Publica, Universidad de Carolina del Sur. 730 Devine Street, Columbia, SC, \\ 29208. E-mail: rpate@gwm.sc.edu \\ ${ }^{3}$ Fisioterapeuta. Especialista en Actividad Física. División de Salud, Fundación FES \\ social, Bogotá; Orise Fellow División de Nutrición y Actividad Física. Centro de \\ Control y Prevención de Enfermedades, Atlanta. E-mail: cbq2@cdc.gov \\ ${ }^{4}$ Médico. Ph. D. Facultad de Medicina, Universidad de los Andes. Bogota, Colombia. \\ E-mail: jduperly@uniandes.edu.co \\ ${ }^{5}$ Médico. MPH. División de Nutrición y Actividad Física. Centro de Control y \\ Prevención de Enfermedades, Atlanta. 4770 Bufford Hwy NE, Mailstop K-46, Atlanta, \\ GA 30341. E-mail: mpratt@cdc.gov
}

Recibido 8 Mayo 2006/Enviado para Modificación 12 Mayo 2006/Aceptado 14 Julio 2006

\section{RESUMEN}

La carga de mortalidad asociada a la inactividad física (IF) no ha sido estimada para los grandes centros urbanos de países en desarrollo.

Objetivo

Calcular la carga de mortalidad por seis enfermedades crónicas no transmisibles (ECNT) asociadas a la IF y estimar el número de muertes potencialmente prevenibles si se reduce la prevalencia de IF en la ciudad.

Métodos La prevalencia de IF obtenida mediante encuestas poblacionales representativas se combinó con datos sobre la mortalidad en adultos mayores de 45 años durante el año 2002. La mortalidad por ECNT atribuible a la IF y la mortalidad prevenible asociada a reducciones del $30 \%$ en la prevalencia de IF se estimaron mediante cálculos del riesgo atribuible poblacional (RAP).

Resultados Una prevalencia de exposición a la IF del $53,2 \%$ se asoció con un RAP de 19,3\% para enfermedad coronaria, 24,2 \% para accidentes cerebro vasculares, 13,8 \% para hipertensión, $21 \%$ para Diabetes Mellitus, 17,9 \% para cáncer de colon y $14,2 \%$ para cáncer de seno. Un $7,6 \%$ de la mortalidad total y un $20,1 \%$ de la mortalidad por ECNT pueden ser atribuibles a la IF. Un $5 \%$ de la mortalidad por ECNT podría evitarse si la prevalencia de IF se reduce en un $30 \%$. 
Conclusiones Una proporción considerable de la mortalidad ocasionada por las ECNT más frecuentes puede atribuirse a los efectos de la IF. Estrategias para la disminución de la IF pueden conllevar a reducciones progresivas de la carga de mortalidad por ECNT en la ciudad.

Palabras Clave: Mortalidad, actividad física, adulto, riesgo (fuente: DeCS, BIREME).

\begin{abstract}
Burden of Mortality Associated to Physical Inactivity in Bogota, Colombia Estimates of the burden of mortality associated to physical inactivity (PI) have not been quantified for large urban centers located in developing countries.

Objectives To estimate the burden of mortality due to six chronic diseases (CDZ) associated to $\mathrm{PI}$ and the number of potentially preventable deaths associated to reductions in the prevalence of PI.

Methods PI exposure prevalence obtained via population surveys was linked to mortality data registered during 2002 among adult (> 45 y) Bogotá residents. The strength of association between $\mathrm{PI}$ and disease-specific mortality was obtained from the literature. Population attributable risk (PAR) was used to calculate the CDZ mortality attributable to $\mathrm{PI}$ and to estimate the number of potentially preventable deaths associated to a $30 \%$ reduction in the prevalence of PI.

Results A 53,2 \% PI exposure prevalence was associated to a PAR of $19,3 \%$ for coronary artery disease, $24,2 \%$ for stroke, 13,8 \% for arterial hypertension, $21 \%$ for Diabetes Mellitus, 17,9 \% for colon cancer and 14,2 \% for breast cancer. An estimated 7,6 \% of all-cause mortality and $20,1 \%$ of CDZ mortality could be attributed to PI. An estimated $5 \%$ of the CDZ mortality could be prevented if $\mathrm{PI}$ prevalence is reduced by $30 \%$.

Conclusion Conservative estimates indicate that a considerable proportion of deaths due to highly prevalent CDZ could be attributed to PI. Strategies to reduce the prevalence of $\mathrm{PI}$ in Bogotá could lead to progressive reductions in the burden of CDZ mortality.
\end{abstract}

Key Words: Mortality, motor activity, adult, risk (source: MeSH, NLM).

$\mathrm{L}$ a carga de mortalidad tanto en países industrializados como en países en desarrollo esta fuertemente influenciada por las muertes ocasionadas por enfermedades crónicas no transmisibles (ECNT). $(1,2)$ El número total de muertes así como las tasas de mortalidad por ECNT son actualmente mayores en los países en desarrollo comparadas con las de países desarrollados (2). La proyección para el año 2020 es que 7 de cada 10 muertes ocurridas en países en desarrollo serán directamente ocasionadas por ECNT (2). Es imperativo destacar que la epidemia de ECNT y sus catastróficas repercusiones en los sistemas de salud no son un problema exclusivo de países 
industrializados, por el contrario, representan una amenaza actual y futura para la salud publica de los países en desarrollo como Colombia (1).

La Organización Mundial para la Salud (OMS) ha hecho recientemente un llamado a los países en desarrollo para que pongan en práctica sistemas de monitoreo y prevención de las ECNT y sus causas subyacentes, con el objetivo de mejorar la salud y la calidad de vida de la población así como de mantener sistemas de salud sostenibles en el futuro $(1,3,4)$. En ciudades como Bogotá esta problemática es evidente. Para la población adulta de Bogotá las ECNT constituyen el principal problema de salud pública debido a los altos costos que éstas generan en relación con morbi-mortalidad prevenible, pérdida de calidad de vida y años de vida productivos y las perdidas financieras que ocasionan al sistema de salud y a la economía de la ciudad (5).

Durante los últimos 30 años abundante evidencia epidemiológica ha sido acumulada sobre la asociación directa entre la inactividad física (IF) y el desarrollo de ECNT (6-10). La IF es actualmente reconocida como un factor de riesgo independiente para el desarrollo de enfermedad cardiovascular $(9,10)$, Diabetes Mellitus tipo $2(9,11)$, síndrome metabólico (12) y algunos tipos de cáncer (9). Adicionalmente, la IF en conjunción con una dieta inadecuada son consideradas como la segunda causa de mortalidad prevenible, particularmente por su contribución al desarrollo de ECNT $(1,13)$.

Los residentes de grandes centros urbanos ubicados en los países en desarrollo experimentan actualmente transiciones epidemiológicas y nutricionales de forma acelerada y en este contexto la IF es un habito prevalente (14-18). En Bogotá, una ciudad de mas de 7 millones de habitantes, uno de cada tres adultos entre 18 y 65 años (36,4 \%) reportan ser inactivos físicamente (16) mientras que la prevalencia de IF en otras grandes ciudades de Latino América es similar $(15,18)$.

Cálculos de la mortalidad atribuible a la IF así como de las posibles muertes prevenibles, si se logra reducir substancialmente la prevalencia de IF, pueden ser utilizados como soporte para la toma de decisiones basadas en evidencia local; conduciendo a la implementación de estrategias de salud pública y cambios en el ambiente construido enfocados hacia la promoción de la actividad física en la ciudad (17,19-21). En consecuencia, los objetivos de este estudio son: 1) estimar la carga de mortalidad por ECNT en adultos residentes en Bogotá ( $>45$ años) atribuible a la IF y 2 ) realizar proyecciones acerca de las muertes por ECNT potencialmente prevenibles si la prevalencia de IF se logra reducir significativamente en esta población.

\section{MÉTODOS}


El presente es un estudio epidemiológico descriptivo de tipo transversal. Las cifras de mortalidad por ENCT registradas durante el año 2002 y los datos recolectados durante 2003 acerca de la prevalencia de IF en la ciudad se combinaron para estimar la carga de mortalidad por ENCT atribuible a la IF en el área urbana de Bogotá.

Datos sobre mortalidad

El número de muertes ocurridas entre adultos mayores de 45 años residentes en Bogotá durante el año 2002 (Enero 1 - Diciembre 31) causadas directamente por enfermedad coronaria (EC), accidentes cerebrovasculares (ACV), hipertensión arterial (HTA), Diabetes Mellitus (DM), cáncer de colon y cáncer de seno se obtuvieron de reportes publicados por la Secretaria Distrital de Salud (5). La selección de estas ECNT como indicadoras de la carga de mortalidad atribuible a la IF siguió varios criterios, entre ellos el reconocimiento de la IF como un factor de riesgo independiente para su desarrollo (6-7,9,20-21). Estas ECNT su ubican entre las 10 causas de muerte mas frecuente para los adultos residentes de Bogotá (5) y ocasionan grandes costos en salud tanto a los que las padecen como a los sistemas de salud y a la economía de la ciudad. Adicionalmente, se tuvo en cuenta la posibilidad de hacer comparaciones con estudios similares que han examinando el impacto de la IF sobre mortalidad por ECNT así como futuras comparaciones locales encaminadas a evaluar objetivamente el impacto de políticas y programas de salud pública $(13,20-22)$.

Los individuos con mayor probabilidad de morir como consecuencia de ECNT (> 45 años) fueron escogidos para los cálculos de mortalidad atribuible a la IF. Los reportes de mortalidad provinieron de datos obtenidos en certificados de obligatorio diligenciamiento para cada defunción en la ciudad, que a su vez son basados en las causas subyacentes (enfermedad desencadenante) de muerte. En el reporte de mortalidad utilizado en esta investigación se utilizó la lista 6/67, versión X de la clasificación internacional de enfermedades publicada por la Organización Panamericana de la Salud (5).

Datos sobre inactividad física

La prevalencia de IF entre los adultos mayores de 45 años residentes en Bogotá se obtuvo por medio de una encuesta realizada durante los meses de Abril y Junio del 2003. Una descripción detallada de los métodos utilizados para la medición de los niveles de actividad física en Bogotá ha sido previamente publicada (16) El instrumento utilizado en la encuesta fue el Cuestionario Internacional de Actividad Física (IPAQ) formato corto (23) el cual fue aplicado a una muestra representativa de la población no institucionalizada de Bogota con un rango de edad entre 15 y 65 años. La validez y reproducibilidad de este instrumento para la medición de los niveles de actividad física en adultos son adecuadas (23). Adicionalmente, el cuestionario fue traducido y adaptado culturalmente para su uso en Colombia (16). Basado en los algoritmos 
de puntuación del cuestionario IPAQ (16), los encuestados fueron categorizados en 3 grupos. Aquellos que reportaron niveles de actividad física consistentes con las recomendaciones actuales (30 minutos al día de actividad física moderada durante 5 o mas días por semana o 20 minutos de actividad física vigorosa 3 o mas días por semana) (6-7) fueron clasificados como activos físicamente. Los individuos que reportaron no haber caminado ni haber participado en actividades físicas de intensidad moderada o vigorosa durante al menos 10 minutos en la semana previa fueron considerados inactivos físicamente. El resto de encuestados que reportaron participar en actividad física pero a niveles inferiores a los recomendados fueron clasificados como insuficientemente activos. Para el presente análisis utilizamos la prevalencia de IF para el grupo de edad entre 50 y 65 años dado que son los individuos inactivos los que presentan mayor riesgo de morbi-mortalidad por ECNT y en quienes se deben enfocar mayores esfuerzos para incrementar los niveles de actividad física $(6,21,24-25)$.

Análisis estadísticos

La carga de mortalidad atribuible a la IF fue estimada usando un método basado en prevalencia (20-21). Primero, la fuerza de asociación entre la IF y la mortalidad específica por cada ECNT se obtuvo de un resumen de riesgos relativos (RR) cuantificados a nivel poblacional en varios estudios epidemiológicos prospectivos previamente publicados (20). En estos estudios el riesgo de morbi-mortalidad por cada una de las ECNT se comparó entre los inactivos y aquellos con niveles de actividad física mayores (Tabla 1). Acto seguido, se estimó el impacto de la IF sobre la mortalidad para cada una de las ECNT estudiadas por medio del calculo del riesgo atribuible poblacional. Esta herramienta epidemiológica combina la asociación (RR) entre la IF y la ECNT y la prevalencia de exposición a la IF en la población estudiada. Para el cálculo del RAP utilizamos la fórmula desarrollada por Levin (26) para dos niveles de exposición:

RAP \% = [prevalencia (RR-1) $] /[1+$ prevalencia (RR-1) $]$

El RAP \% es un método epidemiológico útil para estimar la proporción de un problema de salud pública que es atribuible a un factor de riesgo determinado $(13,21,24,26-27)$. Considerando a la IF como el factor de riesgo causal, el RAP \% permite estimar el porcentaje de muertes atribuibles a la IF y que en teoría se podrían prevenir en la población si este factor de riesgo no estuviera presente. Es por tanto un cálculo teórico pero objetivo de la carga de mortalidad atribuible a una condición específica de riesgo. Es importante anotar que en el RAP \% se ajusta el efecto que otros factores de riesgo causales (cuantificados en los estudios epidemiológicos prospectivos utilizados como referencia) (20) ejercen para el desarrollo de estas enfermedades $(13,21,24,26-$ 27). 
El RAP \% depende principalmente de la prevalencia de exposición a la IF $(21,28)$, dado que la IF es el factor de riesgo modificable (aunque seguramente no erradicable por completo a nivel poblacional) quisimos estimar el número de muertes potencialmente prevenibles si la prevalencia de IF se reduce en un 30\% en la población adulta (29). La reducción en mortalidad que se alcanzaría si se logra este objetivo en salud pública se estimó para cada una de las ECNT estudiadas, en conglomerado para la mortalidad por ECNT y en relación con la mortalidad total en la ciudad durante el periodo estudiado. Todos los análisis se realizaron estratificadamente por grupos de edad y género utilizando SPSS 12 y Excel 2003.

\section{RESULTADOS}

En Bogotá, cerca de un $20 \%$ de la población esta constituida por adultos mayores de 45 años. La mortalidad específica por cada una de las ECNT estudiadas, así como la mortalidad por ECNT en conglomerado y la mortalidad por todas las causas (MT) según estratificaciones por grupos de edad y género es presentada en la Tabla 1. De la MT ocurrida en Bogotá durante el año 2002 entre los adultos mayores de 45 años residentes en la ciudad, un $81,9 \%$ se presentaron en el grupo de mayores de 60 años. Un 37,8 \% De la MT en adultos mayores de 45 años tuvo como causa alguna de las ECNT estudiadas. En el grupo de 45 a 59 años, la EC fue la primera, los ACV la segunda, el cáncer de seno la quinta y la DM la sexta causa de muerte, más frecuente entre los adultos residentes en Bogotá. En el grupo de mayores de 60 años, la EC fue la primera, los ACV la segunda, la DM la cuarta y la HTA la quinta, causas de muerte mas frecuentes. En el conglomerado de MT (24418 defunciones) entre todos los residentes de Bogotá, incluyendo todas las edades, la EC fue la primera, los ACV la segunda, la DM la quinta y la HTA la octava causas de muerte mas frecuentes.

Una prevalencia de IF de 53,2 \% se asoció con un RAP \% de 19,3 \% para la mortalidad por EC, de 24,2 \% para la mortalidad por ACV, de 13,8 \% para la mortalidad por HTA, de $21 \%$ para la mortalidad por T2DM, de 17,9 \% para la mortalidad por cáncer de colon y de 14,2 \% para la mortalidad por cáncer de seno (Tabla 2). Nuestras estimaciones indican que un 20,1 \% de la mortalidad por las seis ECNT estudiadas y un 7,6 \% de la MT en los adultos mayores de 45 años residentes de Bogotá pueden ser atribuidas a los efectos de la IF. El mayor número de muertes atribuibles a la IF se presentó para la EC, los ACV y la DM. 
Tabla 1. Mortalidad ocurrida durante el año 2002 entre los adultos residentes de Bogotá por grupos de edad y género

\begin{tabular}{|c|c|c|c|c|c|c|c|c|c|}
\hline \multirow[b]{2}{*}{ Mortalidad } & \multicolumn{3}{|c|}{ Adultos 45 -59 años } & \multicolumn{3}{|c|}{ Adultos > 60 años } & \multicolumn{3}{|c|}{ Adultos $>45$ años } \\
\hline & $\begin{array}{c}M \\
(\%)\end{array}$ & $\begin{array}{c}F \\
(\%)\end{array}$ & $\begin{array}{c}\mathrm{G} \\
(\% \mathrm{MT})\end{array}$ & $\begin{array}{c}M \\
(\%)\end{array}$ & $\begin{array}{c}F \\
(\%)\end{array}$ & $\begin{array}{c}\mathrm{G} \\
(\% \mathrm{MT})\end{array}$ & $\begin{array}{c}M \\
(\%)\end{array}$ & $\begin{array}{c}F \\
(\%)\end{array}$ & $\begin{array}{c}\mathrm{G} \\
(\% \mathrm{MT})\end{array}$ \\
\hline $\begin{array}{c}\text { Enfermedad } \\
\text { Coronaria }\end{array}$ & $\begin{array}{c}253 \\
(69,3)\end{array}$ & $\begin{array}{c}112 \\
(30,7)\end{array}$ & $\begin{array}{l}365 \\
(11) \\
\end{array}$ & $\begin{array}{l}1200 \\
(49,6)\end{array}$ & $\begin{array}{l}1221 \\
(50,4)\end{array}$ & $\begin{array}{l}2421 \\
(16,7) \\
\end{array}$ & $\begin{array}{l}1453 \\
(52,2)\end{array}$ & $\begin{array}{l}1333 \\
(47,8)\end{array}$ & $\begin{array}{l}2786 \\
(15,7) \\
\end{array}$ \\
\hline $\mathrm{ACV}$ & $\begin{array}{c}121 \\
(45,3)\end{array}$ & $\begin{array}{c}146 \\
(54,7)\end{array}$ & $\begin{array}{c}267 \\
(8,1)\end{array}$ & $\begin{array}{c}640 \\
(41,8)\end{array}$ & $\begin{array}{c}890 \\
(58,2)\end{array}$ & $\begin{array}{c}1530 \\
(10,6)\end{array}$ & $\begin{array}{c}761 \\
(42,3)\end{array}$ & $\begin{array}{l}1036 \\
(57,7)\end{array}$ & $\begin{array}{l}1797 \\
(10,1)\end{array}$ \\
\hline $\begin{array}{c}\text { Hipertensión } \\
\text { Arterial }\end{array}$ & $\begin{array}{c}25 \\
(54,3) \\
\end{array}$ & $\begin{array}{c}21 \\
(45,7)\end{array}$ & $\begin{array}{c}46 \\
(1,4)\end{array}$ & $\begin{array}{c}209 \\
(36,9)\end{array}$ & $\begin{array}{c}357 \\
(63,1)\end{array}$ & $\begin{array}{c}566 \\
(3,9)\end{array}$ & $\begin{array}{c}234 \\
(38,2)\end{array}$ & $\begin{array}{c}378 \\
(61,8)\end{array}$ & $\begin{array}{c}612 \\
(3,5)\end{array}$ \\
\hline $\begin{array}{l}\text { Diabetes } \\
\text { Mellitas }\end{array}$ & $\begin{array}{c}90 \\
(63) \\
\end{array}$ & $\begin{array}{c}54 \\
(37)\end{array}$ & $\begin{array}{c}144 \\
(4,4)\end{array}$ & $\begin{array}{c}375 \\
(43,2)\end{array}$ & $\begin{array}{c}494 \\
(56,8)\end{array}$ & $\begin{array}{l}869 \\
(6)\end{array}$ & $\begin{array}{c}465 \\
(45,9)\end{array}$ & $\begin{array}{c}548 \\
(55,1)\end{array}$ & $\begin{array}{l}1013 \\
(5,7)\end{array}$ \\
\hline $\begin{array}{l}\text { Cáncer } \\
\text { Colon }\end{array}$ & $\begin{array}{c}15 \\
(39,5)\end{array}$ & $\begin{array}{c}23 \\
(60,5)\end{array}$ & $\begin{array}{c}38 \\
(1,2)\end{array}$ & $\begin{array}{c}71 \\
(43)\end{array}$ & $\begin{array}{c}94 \\
(57) \\
\end{array}$ & $\begin{array}{r}165 \\
(1,1)\end{array}$ & $\begin{array}{c}86 \\
(42,4)\end{array}$ & $\begin{array}{c}117 \\
(57,6)\end{array}$ & $\begin{array}{c}203 \\
(1,1)\end{array}$ \\
\hline $\begin{array}{l}\text { Cáncer } \\
\text { Seno }\end{array}$ & $\begin{array}{c}0 \\
(0) \\
\end{array}$ & $\begin{array}{c}145 \\
(100)\end{array}$ & $\begin{array}{c}145 \\
(4,4)\end{array}$ & $\begin{array}{c}1 \\
(0,6)\end{array}$ & $\begin{array}{c}162 \\
(99,4)\end{array}$ & $\begin{array}{c}163 \\
(1,1)\end{array}$ & $\begin{array}{c}1 \\
(0,3)\end{array}$ & $\begin{array}{c}307 \\
(99,7)\end{array}$ & $\begin{array}{c}308 \\
(1,7)\end{array}$ \\
\hline $\begin{array}{l}\text { Mortalidad } \\
\text { por ECNT }\end{array}$ & $\begin{array}{c}504 \\
(50,1) \\
\end{array}$ & $\begin{array}{c}501 \\
(49,9) \\
\end{array}$ & $\begin{array}{r}1005 \\
(30,5) \\
\end{array}$ & $\begin{array}{c}2496 \\
(43,7) \\
\end{array}$ & $\begin{array}{l}3218 \\
(56,3) \\
\end{array}$ & $\begin{array}{l}5714 \\
(39,5) \\
\end{array}$ & $\begin{array}{l}3000 \\
(44,6) \\
\end{array}$ & $\begin{array}{l}3719 \\
(55,4) \\
\end{array}$ & $\begin{array}{l}6719 \\
(37,8) \\
\end{array}$ \\
\hline MT & $1799(54,6)$ & $\begin{array}{l}1497 \\
(45,4) \\
\end{array}$ & $\begin{array}{l}3296 \\
(100)\end{array}$ & $\begin{array}{l}6914 \\
(47,8) \\
\end{array}$ & $\begin{array}{l}7552 \\
(52,2) \\
\end{array}$ & $\begin{array}{c}14466 \\
(100)\end{array}$ & $\begin{array}{l}8713 \\
(49,1) \\
\end{array}$ & $\begin{array}{l}9049 \\
(50,9) \\
\end{array}$ & $\begin{array}{c}17762 \\
(100)\end{array}$ \\
\hline
\end{tabular}

M, masculino; F, femenino; G, general; ACV, Accidentes cerebrovasculares; MT, Mortalidad por todas las causas; ECNT, Enfermedades crónicas no transmisibles

Tabla 2. Mortalidad atribuible a la inactividad física durante 2002 y prevenible si se reduce en un $30 \%$ su prevalencia

\begin{tabular}{|c|c|c|c|c|c|c|c|c|c|}
\hline \multirow{3}{*}{ Patologías } & \multirow{3}{*}{$\begin{array}{c}\mathrm{RR} \\
(95 \% \mathrm{Cl})\end{array}$} & \multirow{3}{*}{ RAP \%* } & \multirow{2}{*}{\multicolumn{3}{|c|}{$\begin{array}{c}\text { Mortalidad Atribuible } \\
\text { a IF } \\
\text { Años }\end{array}$}} & \multirow{3}{*}{ RAP $\%{ }^{\dagger}$} & \multirow{2}{*}{\multicolumn{3}{|c|}{$\begin{array}{c}\text { Mortalidad Atribuible } \\
\text { a IF } \\
\text { Años }\end{array}$}} \\
\hline & & & & & & & & & \\
\hline & & & $45-59$ & $>60$ & $>45$ & & $45-59$ & $>60$ & $>45$ \\
\hline $\begin{array}{l}\text { Enfermedad } \\
\text { Coronaria }\end{array}$ & $\begin{array}{c}1,45 \\
(1,38-1,54)\end{array}$ & 19,3 & & & & 14,4 & 52 & 347 & 399 \\
\hline $\mathrm{ACV}$ & $\begin{array}{c}1,60 \\
(1,42-1,80)\end{array}$ & 24,2 & 65 & 370 & 435 & 18,3 & 49 & 279 & 328 \\
\hline $\begin{array}{l}\text { Hipertensión } \\
\text { Arterial }\end{array}$ & $\begin{array}{c}1,30 \\
(1,16-1,46)\end{array}$ & 13,8 & 6 & 78 & 84 & 10,1 & 5 & 57 & 62 \\
\hline $\begin{array}{l}\text { Diabetes } \\
\text { Mellitas }\end{array}$ & $\begin{array}{c}1,50 \\
(1,37-1,63)\end{array}$ & 21 & 30 & 183 & 213 & 15,7 & 15 & 17 & 159 \\
\hline $\begin{array}{l}\text { Cáncer } \\
\text { Colon }\end{array}$ & $\begin{array}{c}1,41 \\
(1,31-1,53)\end{array}$ & 17,9 & 7 & 30 & 37 & 13,3 & 5 & 22 & 27 \\
\hline $\begin{array}{l}\text { Cáncer } \\
\text { Seno }\end{array}$ & $\begin{array}{c}1,31 \\
(1,23-1,38)\end{array}$ & 14,2 & 21 & 23 & 44 & 10,4 & 15 & 17 & 32 \\
\hline $\begin{array}{l}\text { Mortalidad } \\
\text { por ECNT }\end{array}$ & 1 & - & 199 & 1151 & 1350 & - & 148 & 859 & 1007 \\
\hline $\begin{array}{l}\text { \% Mortalidad } \\
\text { por ECNT }\end{array}$ & - & - & 19,8 & 20,1 & 20,1 & - & 14,7 & 15 & 15 \\
\hline$\% \mathrm{MT}$ & - & - & 6 & 8 & 7,6 & - & 4,5 & 5,9 & 5,7 \\
\hline
\end{tabular}

RR, Riesgo relativo; IC, Intervalo de confianza; RAP, Riesgo atribuible poblacional; IF, Inactividad física; ACV, Accidentes cerebrovasculares; MT, Mortalidad por todas las causas; ECNT, Enfermedades crónicas no transmisibles; * Estimativos calculados usando una prevalencia de exposición a la IF de $53,2 \% ;{ }^{\dagger}$ Estimativos calculados usando una prevalencia de exposición a la IF de 37,2 \% (meta de reducción del 30\%)

Los resultados de los modelos desarrollados para estimar la mortalidad prevenible asociada a una reducción de la IF de un $30 \%$ se presentan en la Tabla 2. La MT y la mortalidad por ECNT entre los adultos mayores de 45 años 
residentes en Bogotá se podrían reducir en un 1,9 \% y 5,1 \% respectivamente si la prevalencia de IF en este grupo se reduce a un $37,2 \%$.

El número de muertes por cada una de las ECNT y la mortalidad por ECNT en conglomerado atribuibles a una prevalencia de IF del 53,2 \% y las que se podrían evitar si la prevalencia de IF se reduce a un 37,2 \% son comparadas en la Figura 1. La reducción en mortalidad asociada a disminuciones en la prevalencia de la IF fue mayor para la EC, los ACV y la DM.

Figura 1. Mortalidad por enfermedades crónicas no transmisibles (ECNT) atribuibles a la inactividad física (IF) y mortalidad prevenible si la prevalencia de IF se reduce en un $30 \%$

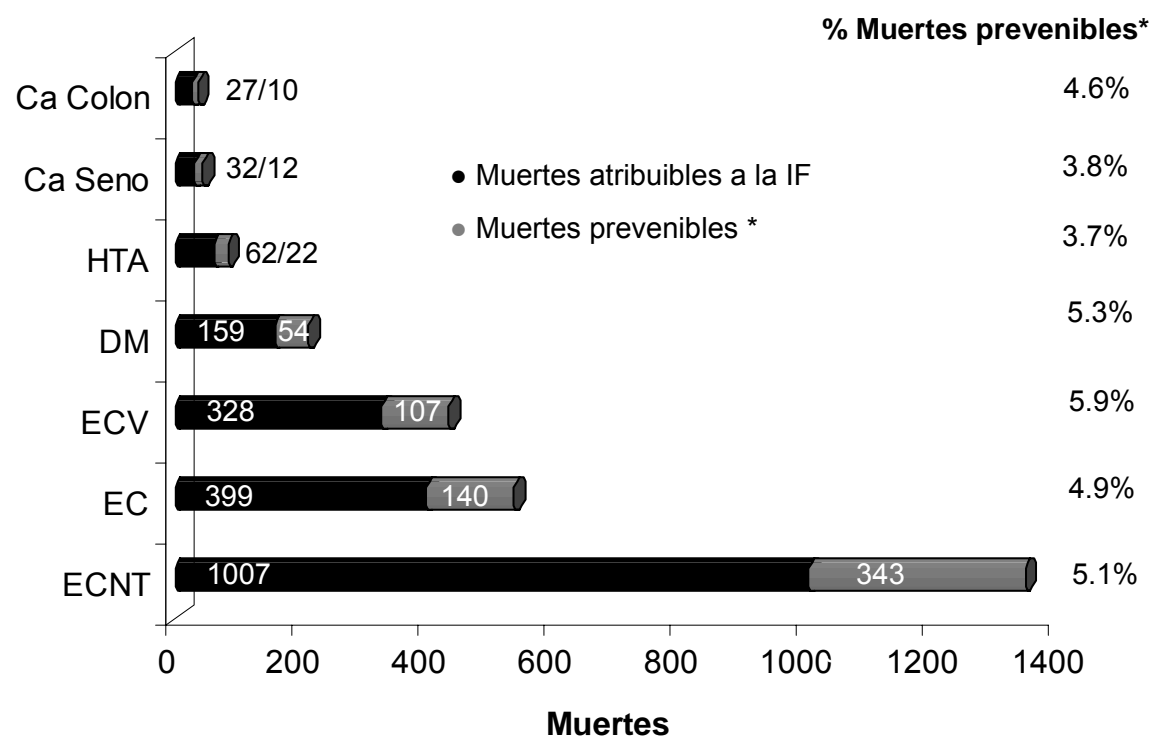

"Ca (Cancer); HTA (hipertensión); DM (Diabetes Mellitus); ECV (enfermedad cerebrovascular); EC (Enfermedad coronaria)

\section{DISCUSION}

De nuestro conocimiento, este es el primer estudio que intenta estimar la carga de mortalidad por enfermedades crónicas no transmisibles asociada a la inactividad física en un gran centro urbano de Latinoamérica. Nuestras estimaciones, mas bien conservadoras, indican que una proporción considerable (una de cada cinco) de las muertes por ECNT ocurridas en los adultos mayores de 45 años residentes en Bogotá pueden ser atribuidas a los efectos de la IF. Adicionalmente, un $5 \%$ de la mortalidad por ECNT y un $2 \%$ de la MT en esta población son potencialmente prevenibles si metas en salud pública enfocadas a 
reducir en un tercio la prevalencia de adultos inactivos físicamente son alcanzadas en la ciudad.

En el contexto del presente estudio constatamos que en Bogota las ECNT relacionadas con procesos de arteriosclerosis y daño en el sistema vascular constituyen la principal causa de muerte prematura entre adultos de 45 a 59 años y de muerte en adultos mayores de 60 años. Esta es una tendencia epidemiológica establecida tanto en los países industrializados como en la gran mayoría de los países en desarrollo $(1-2,5,15,20,22)$. Dado que la IF es un factor de riesgo establecido e independiente para morbi-mortalidad por EC, ACV y DM $(6,7,10)$, no sorprende encontrar que una gran proporción de las muertes por estas enfermedades ocurridas en la ciudad (entre un 20 y $25 \%$ ) sean atribuibles a la IF y por tanto potencialmente prevenibles si los niveles de IF son reducidos en la población. En consecuencia la prevención de enfermedades cardiovasculares (EC, ACV) y metabólicas (DM, síndrome metabólico, obesidad) debe constituirse en una prioridad de salud pública en la ciudad. En este contexto, la implementación de estrategias de prevención primaria y secundaria encaminadas a incrementar los niveles de actividad física y adoptar hábitos de vida saludable debe ser fortalecida en un grado paralelo al impacto que estos factores de riesgo ejercen sobre la salud de la población adulta en Bogotá (10,17-18,30).

Nuestros cálculos indican que un $5 \%$ de la mortalidad por ECNT y un $2 \%$ de la mortalidad por todas las causas podrían prevenirse si la prevalencia de adultos inactivos físicamente se reduce considerablemente en la ciudad. Investigaciones previas en naciones industrializadas han estimado la reducción en mortalidad asociada con cambios positivos en los niveles de actividad física en la población. Usando datos de mortalidad y prevalencia de IF durante 1988 en Estados Unidos, Powell et al (21) calcularon una importante reducción del $5.4 \%$ en la mortalidad por ECNT y del $1,5 \%$ en la mortalidad por todas las causas, asociadas al cumplimiento de los objetivos en salud pública sobre actividad física para el año 2000 trazados por el Departamento de Salud de ese país $(21,29)$. Cálculos realizados en naciones experimentando rápidos procesos de industrialización y de transición nutricional y epidemiológica también indican que un porcentaje significativo $(6,8 \%)$ de la mortalidad en adultos es potencialmente prevenible si la IF se reduce (22). Bajo esta perspectiva nuestros resultados indican que: 1) en Bogotá, la carga de mortalidad atribuible a la IF es en la actualidad similar a la de regiones industrializadas en donde la reducción de la IF es considerada una prioridad de salud pública $(18,29)$; 2) en consecuencia es imperativo establecer sistemas para el monitoreo epidemiológico de la IF en nuestra población (4); y 3) es prioritario establecer objetivos claros en salud pública encaminados a la reducción de este factor de riesgo, acompañados de la implementación de políticas y estrategias que permitan alcanzarlos $(1,17,18,29)$. 
Además del gran impacto de la IF sobre la morbi-mortalidad es importante resaltar su efecto sobre otros indicadores de salud y calidad de vida. Estos incluyen la pérdida prematura de años de vida y de años de vida productiva por causa de enfermedad (DALYs), el impacto sobre calidad de vida (QALYs) (1) y los costos financieros directos e indirectos asociados a estos indicadores. Varios estudios económicos han cuantificado el costo en salud asociado a la IF. En Canadá se estimó que un 2,6 \% de los costos totales en salud durante 2001 fueron causados por la IF (20) mientras que en Estados Unidos fue de un 2,4 \% durante 1994 (31). En este país, un 13,1 \% de los costos totales relacionados con el manejo de la enfermedad cardiovascular durante 1996 (41,3 billones de dólares) se atribuyeron a la IF (32). Así mismo en Nueva Zelanda, un 2,5 \% de los costos totales en salud del país se asociaron a la obesidad, una condición estrechamente relacionada con la IF (33). El presente estudio no intentó estimar el impacto económico de la IF, en parte por la dificultad de consecución de los costos directos e indirectos relacionados con las seis ECNT estudiadas. Sin embargo, se puede especular que los gastos en salud asociados a la IF en la ciudad serán similares a aquellos encontrados en otros países ya que las cargas de mortalidad asociada a la IF son comparables. Teniendo como referencia el presupuesto del Fondo Financiero Distrital de Salud establecido por el consejo de Bogotá para el 2002 (34) se podría estimar que unos 15 mil millones de pesos (2,5 \% del presupuesto) corresponden a los gastos ocasionados por la IF. Futuros análisis económicos estimando el costo en salud de la IF son necesarios para confirmar estas hipótesis.

Recientemente, en Bogotá se han implementado cambios significativos con respecto a políticas de transporte, recreación, estrategias de promoción de actividad física y cambios en el ambiente construido, que pueden contribuir a reducir la prevalencia de IF (35-36). Estos cambios incluyen entre otros, el fortalecimiento del sistema de transporte público masivo (Transmilenio), (35) el incentivo a otros medios de transporte alternativos como la bicicleta por medio de la construcción de $300 \mathrm{Km}$ de "Ciclorutas" a lo largo y ancho de la ciudad (35-36), la habilitación y recuperación de espacios públicos y vías peatonales (35), el uso selectivo de calles y vías de transporte para la recreación y práctica de actividad física durante los domingos y días festivos (Ciclovía) y la implementación de programas de actividad física y recreación a lo largo del sistema de parques y Ciclovías de la ciudad (Recreovía), entre otros. La costoefectividad de estas y otras intervenciones en términos de su impacto directo sobre los niveles de actividad física de los habitantes de la ciudad e indirectamente sobre la reducción en morbi-mortalidad por ECNT debe ser una prioridad de investigación para el futuro en este campo (19,37-38); Sin embargo, los resultados del presente estudio brindan información importante para los funcionarios en capacidad de tomar decisiones dentro de los sistemas de salud y gobierno locales y en los departamentos de planeación y de 
transporte urbanos con respecto a lo que debe constituirse en prioridades y políticas de salud pública. Dentro de estas organizaciones, la promoción permanente y sostenible de estrategias encaminadas a la reducción de la IF a nivel poblacional debe seguir jugando un rol primordial.

Como fortalezas de esta investigación se encuentran el uso de datos actuales y representativos con respecto a la prevalencia de exposición a la IF y a la mortalidad por ECNT, recolectada a su vez con instrumentos validos. Se usaron estimativos de riego (RR) representando la fuerza de asociación entre la mortalidad por las ECNT estudiadas y la IF, los cuales fueron derivados de estudios epidemiológicos prospectivos, diseñados para estudiar la mencionada asociación. Finalmente, quisimos ir más allá del cálculo de la mortalidad atribuible a la IF y estimamos la carga de mortalidad prevenible asociada a reducciones plausibles en la prevalencia de IF para enfocarnos en prácticas “reales” y objetivos alcanzables en salud pública.

Como limitaciones del presente estudio se pueden mencionar la imposibilidad de tener datos acerca de mortalidad e IF en la misma población durante el mismo año. Sin embargo, la diferencia de tiempo entre el factor etiológico estudiado y la mortalidad por ECNT, fue menor a 1 año, haciendo improbables cambios dramáticos en la prevalencia de IF durante este periodo. Adicionalmente, se sabe que los niveles de actividad física varían con el tiempo en los individuos y por tanto su riesgo de ECNT cambia (39). El efecto de este fenómeno es el de atenuar las estimaciones del riesgo de morbi-mortalidad por ECNT. Además, la disminución del riesgo de ECNT asociada a la práctica regular de AF es mediada por su efecto en la regulación de variables biológicas como el perfil lipidico, la sensibilidad a la insulina o la mejoría en la función y la capacidad cardiovascular $(7,9)$. Todos estos son puntos intermedios en la cadena de asociación causal entre la AF y varias ECNT que, típicamente, no son detectados en los estimativos de asociación obtenidos por medio de estudios epidemiológicos. Estas consideraciones deben ser tenidas en cuenta en la interpretación de nuestros resultados que son posiblemente conservadores (21).

En conclusión, una proporción considerable de las muertes registradas durante el año 2002 en los adultos mayores de 45 años y ocasionadas por las ECNT más frecuentes en la ciudad pueden ser atribuidas a los efectos de la IF. Si se cumplen metas específicas en salud pública encaminadas a reducir substancialmente la prevalencia de IF en la población adulta, la carga de mortalidad por ECNT en la ciudad puede ser atenuada progresivamente de manera significativa. Investigaciones acerca del impacto en salud y económico de la IF pueden brindar fundamentos lógicos adicionales para destinar recursos hacia la implementación y sostenimiento de estrategias y políticas publicas de promoción de actividad física en la ciudad • 
Agradecimientos. Al Dr. Joan Mendivil Medina por su colaboración en la edición del manuscrito.

\section{REFERENCIAS}

1. World Health Organization. Preventing chronic diseases: a vital investment. WHO global report; 2005.

2. Boutayeb A, Boutayeb S. The burden of non communicable diseases in developing countries. Int J Equity Health 2005 January 14; 4(1): 2.

3. Epping-Jordan JE, Galea G, Tukuitonga C, Beaglehole R. Preventing chronic diseases: taking stepwise action. Lancet 2005 November 5; 366(9497): 1667-71.

4. Strong KL, Bonita R. Investing in surveillance: a fundamental tool of public health. Soz Praventivmed 2004; 49(4): 269-75.

5. Cardona JF. Boletín de Estadísticas Julio-Diciembre 2003.Secretaria Distrital de Salud de Bogota; 2003.

6. Pate RR, Pratt M, Blair SN, Haskell WL, Macera CA, Bouchard C, et al. A recommendation from the Centers for Disease Control and Prevention and the American College of Sports Medicine. JAMA 1995; 273(5): 402-7.

7. U.S.Department of Health and Human Services. Physical Activity and Health: A Report of the Surgeon General. Atlanta: USDHSS/CDC; 1996.

8. Katzmarzyk PT, Janssen I. The economic costs associated with physical inactivity and obesity in Canada: an update. Can J Appl Physiol 2004 February; 29(1): 90-115.

9. Eyre H, Kahn R, Robertson RM, Clark NG, Doyle C, Hong Y, et al. Preventing cancer, cardiovascular disease, and diabetes: a common agenda for the American Cancer Society, the American Diabetes Association, and the American Heart Association. Circulation 2004 June 29; 109(25): 3244-55.

10. Thompson PD, Buchner D, Pina IL, Balady GJ, Williams MA, Marcus BH et al. Exercise and physical activity in the prevention and treatment of atherosclerotic cardiovascular disease: a statement from the Council on Clinical Cardiology (Subcommittee on Exercise, Rehabilitation, and Prevention) and the Council on Nutrition, Physical Activity, and Metabolism (Subcommittee on Physical Activity). Circulation 2003 June 24; 107(24): 3109-16.

11. Knowler WC, Barrett-Connor E, Fowler SE, Hamman RF, Lachin JM, Walker EA et al. Reduction in the incidence of type 2 diabetes with lifestyle intervention or metformin. N Engl J Med 2002 February 7; 346(6): 393-403.

12. Laaksonen DE, Lakka HM, Salonen JT, Niskanen LK, Rauramaa R, Lakka TA. Low Levels of Leisure-Time Physical Activity and Cardiorespiratory Fitness Predict Development of the Metabolic Syndrome. Diabetes Care 2002 September 1; 25(9): 1612-8.

13. Mokdad AH, Marks JS, Stroup DF, Gerberding JL. Actual causes of death in the United States, 2000. JAMA 2004 March 10; 291(10): 1238-45.

14. Popkin BM. The nutrition transition: an overview of world patterns of change. Nutr Rev 2004 July; 62(7 Pt 2): S140-S143.

15. Rivera JA, Barquera S, Campirano F, Campos I, Safdie M, Tovar V. Epidemiological and nutritional transition in Mexico: rapid increase of non-communicable chronic diseases and obesity. Public Health Nutr 2002 February; 5(1A): 113-22. 
16. Gomez LF, Duperly J, Lucumi DI, Gamez R, Venegas AS. Physical activity levels in adults living in Bogota (Colombia): prevalence and associated factors. Gac Sanit 2005 May; 19(3): 206-13.

17. Pratt M, Jacoby ER, Neiman A. Promoting physical activity in the Americas. Food Nutr Bull 2004 June; 25(2): 183-93.

18. Salinas J, Vio F. Promoting health and physical activity in Chile: a policy priority. Rev Panam Salud Publica 2003 October; 14(4): 281-8.

19. Hill JO, Sallis JF, Peters JC. Economic analysis of eating and physical activity: a next step for research and policy change. Am J Prev Med 2004 October; 27(3 Suppl): 111-6.

20. Katzmarzyk PT, Janssen I. The economic costs associated with physical inactivity and obesity in Canada: an update. Can J Appl Physiol 2004 February; 29(1): 90-115.

21. Powell KE, Blair SN. The public health burdens of sedentary living habits: theoretical but realistic estimates. Med Sci Sports Exerc 1994 July; 26(7): 851-6.

22. He J, Gu D, Wu X, Reynolds K, Duan X, Yao C et al. Major causes of death among men and women in China. N Engl J Med 2005 September 15; 353(11): 112434.

23. Craig CL, Marshall AL, Sjostrom M, Bauman AE, Booth ML, Ainsworth BE et al. International physical activity questionnaire: 12-country reliability and validity. Med Sci Sports Exerc 2003 August; 35(8): 1381-95.

24. Bauman A. Use of population attributable risk (PAR) in understanding the health benefits of physical activity. Br J Sports Med 1998 December; 32(4): 279-80.

25. Macera CA, Powell KE. Population attributable risk: implications of physical activity dose. Med Sci Sports Exerc 2001 June; 33(6 Suppl): S635-S639.

26. Levin ML. The occurrence of lung cancer in man. Acta Un Inter Cancer 1953; 9(3): 531-41.

27. Rockhill B, Newman B, Weinberg C. Use and misuse of population attributable fractions. Am J Public Health 1998 January; 88(1): 15-9.

28. Szklo M, Nieto JF. Epidemiology: Beyond the basics. 1st Ed. Gaithersburg, Maryland: Aspen Publishers; 2000.

29. U.S.Department of Health and Human Services. Healthy People 2010 (2nd edition). Washington,D.C.: U.S. Government Printing Office; 2000.

30. Duperly J, Lobelo F. Estilo de vida saludable: un reto que vale la pena. Revista Colombiana de Cardiologia 2004; 11(3): 139-40.

31. Colditz GA. Economic costs of obesity and inactivity. Med Sci Sports Exerc 1999 November; 31(11 Suppl): S663-S667.

32. Wang G, Pratt M, Macera CA, Zheng ZJ, Heath G. Physical activity, cardiovascular disease, and medical expenditures in U.S. adults. Ann Behav Med 2004 October; 28(2): 88-94.

33. Swinburn B, Ashton T, Gillespie J, Cox B, Menon A, Simmons D et al. Health care costs of obesity in New Zealand. Int J Obes Relat Metab Disord 1997 October; 21(10): 891-6.

34. Alcaldía Mayor de Bogota. Presupuesto Anual de Rentas e Ingresos y de Gastos e Inversiones del Distrito Capital para el año 2002 [Internet]. Disponible en http://www.alcaldiabogota.gov.co/sisjur/. Consultado Abril del 2006.

35. Cervero R. Progressive transport and the poor: Bogota's bold steps forward. Access 2005; 27(fall): 24-30. 
36. Gomez LF, Sarmiento OL, Lucumi DI, Espinosa G, Forero R, Bauman A. Prevalence and Factors Associated with Walking and Bicycling for Transport Among Young Adults in Two Low-Income Localities of Bogotá, Colombia. Journal of Physical Activity and Health 2005; 2(4): 445-59.

37. Matsudo V, Matsudo S, Andrade D, Araujo T, Andrade E, de Oliveira LC, et al. Promotion of physical activity in a developing country: the Agita Sao Paulo experience. Public Health Nutr 2002 February; 5(1A): 253-61.

38. Wang G, Macera CA, Scudder-Soucie B, Schmid T, Pratt M, Buchner D. Cost effectiveness of a bicycle/pedestrian trail development in health promotion. Prev Med 2004 February; 38(2): 237-42.

39. Andersen LB. Relative risk of mortality in the physically inactive is underestimated because of real changes in exposure level during follow-up. Am J Epidemiol 2004 July 15; 160(2): 189-95. 